\title{
Development and course of chronic pain over four years in the general population: The HUNT pain study
}

Tormod Landmark ${ }^{1,2^{*}}$,Ola Dale ${ }^{3,4}$, Pål Romundstad ${ }^{5}$, Astrid Woodhouse ${ }^{2,3,5}$, Stein Kaasa ${ }^{6,7,8}$ Petter C. Borchgrevink ${ }^{2,3}$

${ }^{1}$ Department of Psychology, Norwegian University of Science and Technology, Trondheim, Norway

${ }^{2}$ National Competence Centre for Complex Symptom Disorders, St. Olav's Hospital, University Hospital of Trondheim, Trondheim, Norway

${ }^{3}$ Department of Circulation and Medical Imaging, NTNU - Norwegian University of Science and Technology, Trondheim, Norway

${ }^{4}$ Department of Research, St. Olav's Hospital, University Hospital of Trondheim, Norway

${ }^{5}$ Department of Public Health and Nursing, NTNU - Norwegian University of Science and Technology, Trondheim, Norway

${ }^{6}$ Department of Oncology, Oslo University Hospital, Oslo, Norway

${ }^{7}$ Department of Clinical Medicine, Faculty of Medicine, University of Oslo, Oslo, Norway

${ }^{8}$ Department of Cancer Research and Molecular Medicine, Norwegian University of Science and Technology, Trondheim, Norway

\section{"Corresponding author:}

Tormod Landmark

NTNU - Norwegian University of Science and Technology

Department of Psychology

NO-7491 Trondheim

Norway

Tel + 4772826332

E-mail: tormod.landmark@ntnu.no

Original article

Funding source: The HUNT pain study was funded by the Research Council of Norway.

Conflicts of interest: None declared

Significance: The development and recovery of chronic pain is highly dependent on previous pain. The prognosis of chronic pain may be predicted well when considering its duration in combination with other clinical, social and psychological factors. Targeting modifiable prognostic factors may be particularly important for newly developed chronic pain. 


\section{Abstract:}

Background: Epidemiological studies of chronic pain frequently report high prevalence estimates. However, there is little information about the development and natural course of chronic pain. Methods: We followed a random sample of participants from a population-based study (HUNT 3) with annual measures over four years. Results: Among those without chronic pain at baseline, the probability of developing moderate to severe chronic pain (cumulative incidence) during the first year was $5 \%$, a pain status that was maintained among $38 \%$ at the second follow-up. The probability of developing chronic pain diminished substantially for those who maintained a status of no chronic pain over several years. Subjects with moderate to severe chronic pain at baseline had an $8 \%$ probability of recovery into no chronic pain, a status that was maintained for $52 \%$ on the second follow-up. The probability of recovery diminished substantially as a status of chronic pain was prolonged for several years. Pain severity, widespread pain, pain catastrophizing, depression and sleep were significant predictors of future moderate to severe chronic pain, both among subjects with and without chronic pain at baseline. Conclusion: These findings suggest that the prognosis is fairly good after a new onset of chronic pain. When the pain has lasted for several years, the prognosis becomes poor. The same social and psychological factors predict new onset and the prognosis of chronic pain.

Significance: The development and recovery of chronic pain is highly dependent on previous pain. The prognosis of chronic pain may be predicted well when considering its duration in combination with other clinical, social and psychological factors. Targeting modifiable prognostic factors may be particularly important for newly developed chronic pain. 


\section{Introduction:}

Chronic pain is highly prevalent, affecting about $30 \%$ of the adult population, and has detrimental consequences in terms of reduced work capacity, increased health care utilization and declined health and functioning (Landmark et al., 2013a; Steingrimsdottir et al., 2017). We have limited information on the development and natural course of chronic pain. Longitudinal epidemiological studies are required for this, but previous studies have only used measures at two occasions with long follow-up intervals (Elliott et al., 2002; Eriksen et al., 2004). This limits conclusions regarding the pace at which transitions are made and the effect of previous pain on these transitions.

The International Association for the Study of Pain (IASP) has defined chronic pain as pain lasting for more than three months, but proposed a cutoff at 6 months as more appropriate for research purposes (Merskey and Bogduk 1994). This broad definition is likely to include many subjects minimally afflicted by pain. Therefore, it is also of interest to identify those likely to have chronic pain of clinical relevance. For the upcoming ICD-11 classification of chronic pain, an IASP task force proposed either pain related intensity, distress or impairment as additional measures of severity (Treede et al., 2015).

Chronic pain may be the result of ongoing pathological processes that do not heal, or it may be pain that persist beyond the healing of an initial tissue damage. Often the etiology is unknown (Treede et al., 2015). It is widely recognized that multiple causal factors are involved when pain persists (Chapman et al., 2008; Finan et al., 2013; Gerrits et al., 2015). The most common prognostic factors such as higher age, baseline pain, mental health complaints and adverse coping, are shared for various chronic pain conditions (Mallen et al., 2007). Also, an underlying genetic factor has been shown to be shared across conditions (Williams et al., 2010). More often than not, subjects who experience chronic pain report pain at multiple sites (Carnes et al., 2007; Schmidt and Baumeister 2007), and the number of sites is associated with negative outcomes and prognosis (Kamaleri et al., 2008; Von Korff and Miglioretti 2005). These are arguments for studying chronic pain as a generic entity, not divided into different pain conditions.

In the current study, annual measures of chronic pain were obtained over four years in a populationbased sample. The main aim was to characterize the development and course of chronic pain in the general population. More precisely, we intended to investigate the probability of changing from a state of not having chronic pain to having chronic pain (cumulative incidence) and vice versa (recovery), and to take into account previous chronic pain on these transitions. Secondly, we wanted to identify predictors of future chronic pain, both among those without and those with chronic pain at baseline. 


\section{Methods}

\subsection{Sample and procedure}

The HUNT study includes three health surveys, conducted in 1984-1986 (HUNT 1), 1995-1997 (HUNT 2) in and 2006 -2008 (HUNT 3), of the total population aged 20 years and more in Nord -Trøndelag County, Norway. In HUNT 3, 94194 subjects were invited to participate, and a total of 50827 (54\%) attended the study. The demographics of the HUNT population are similar to the average of the Norwegian population, except from a lower average income and education level. The population is stable with a net out migration of $0.3 \%$, and homogenous ( $97 \%$ Caucasian), making it suitable for epidemiological studies (Krokstad et al., 2013).

After approval by the Regional Committee for Medical and Health Research Ethics Central-Norway, a random sample of participants in the HUNT 3 study from the municipalities of Levanger and Verdal $(\mathrm{N}=6419)$ were invited to participate in a sub study of pain (the HUNT pain study). Among them, 4782 (75\%) agreed to participate and to answer postal questionnaires every three months over one year (five questionnaires in total) and thereafter one annual questionnaire for the following three years. At the end of the four year follow up 3405 subjects ( $71 \%$ of respondents and $53 \%$ of those initially invited) returned the last annual questionnaire which was mailed in the period September 2011 to February 2012. The sample size was determined by assuming an incidence of $2 \%$, which required a total of 8000 person-years to obtain $95 \%$ confidence intervals to run from $1.7 \%$ to $2.3 \%$. The flow of participants thorough the study and the different analyses is outlined in figure 1.

-Please insert figure1 about here-

\subsection{Measures}

Chronic pain was identified by two questions. The question, "Do you have bodily pain which has lasted for more than 6 months?" was constructed to meet the IASP proposal for a case definition (Merskey and Bogduk 1994). In addition, a one-week recall of pain intensity ranging from no pain to very mild, mild, moderate, severe and very severe pain was included to divide between subjects with minimal and clinically important chronic pain. The latter is included in the SF-8 and SF-36 health surveys which have shown robust psychometric properties (Ware et al., 2001). A cutoff between mild and moderate on the scale has been demonstrated to successfully identify individuals with pain of a more complex nature (Jensen et al., 2004). The combination of the measures into pain lasting more than six months and at least moderate intensity corresponds highly with longitudinal recordings of pain and is well suited for identifying subjects with chronic pain in the general population (Landmark et al., 2012). 
A body map including predefined layman labels of body areas was used to ascertain cases meeting the American College of Rheumatology's 1990 criteria for widespread pain which states that pain should be present in both the left and right side of the body as well as above and below the waist, and additional axial skeletal pain (Wolfe et al., 1990). This definition does not specify which body areas should be included in the assessment. However, we used the same areas as was described in a recent evaluation of the Widespread Pain Index (WPI), a similar instrument (Wolfe et al., 2016). The areas were: Shoulders, arms, elbows, wrists, hands, calves, hips, thighs, knees, neck, and upper and lower back. We also included ankles/feet in our definition, although they are not included in the WPI.

The two-item version of the Coping Strategies Questionnaire's Catastrophizing scale was used to measure participants' subjective evaluation of helplessness and threat when experiencing pain (Jensen et al., 2003). The items are scored on a 6 point scale ranging from "never do" to "always do that". To identify cases, a cutoff point at $\geq 3$ of the mean aggregated scale was chosen. This was equal to the $70^{\text {th }}$ percentile of the sample with chronic pain at baseline. Moreover, 3 was the midpoint of the scale and had the anchoring "sometimes do that".

The short version of the Mental health inventory (MHI-5) was included as a measure of psychological distress (Berwick et al., 1991). The MHI-5 consists of the five items best representing the full-length version and covers four domains (depression, anxiety, positive affect and behavioral / emotional control). Scoring is designed to produce a scale ranging from 0 to 100 with higher scores indicating better mental health. It has shown good screening properties, in particular for depression, with suggested optimal cutoff point at $\leq 60$ in both general populations and among pain patients (Berwick et al., 1991; Choi et al., 2014; Rumpf et al., 2001).

Global sleep dissatisfaction was measured by the question:" During the past week, have you had trouble sleeping?" The item is a 4 point scale ranging from "not at all" to "a little", "some" and "very much". Cutoff was set between not at all and a little. Similar one-item measures of global sleep dissatisfaction has previously been shown to be a good indicator of the presence of a sleep disorder in the general population (Ohayon and Paiva 2005).

Information on education was obtained from Statistics Norway, which provided data from the National Education database (NUDB). The highest attained level of education was classified into three levels; as primary, secondary or tertiary education. Weight and height were measured at the HUNT 3 screening station. Body-mass Index (BMI) was calculated as weight divided by height squared. Smoking was measured in the HUNT 3 questionnaire and the data was classified as nonsmoker, previous smoker and current smoker. 


\subsection{Statistical analyses}

The probability of transition (changes) between chronic pain states are given as percentages and were calculated by tabulating the chronic pain reports (no, mild or moderate to severe) on every follow up occasion by the chronic pain reports on its previous occasions. Results are displayed in a figure showing the probability of maintaining the baseline chronic pain status from each occasion to the next, and the probability of changing into another category as the baseline category was maintained. For clarity and simplicity, we then dichotomized chronic pain as outcome measure into no or mild vs moderate or severe. We then calculated the number of occasions with moderate to severe chronic pain during the follow up period. These analyses were stratified by baseline chronic pain (no, mild or moderate to severe). Only subjects with complete data were included in the descriptive analyses of pain transition and stability.

Prospective associations between baseline characteristics and reporting of moderate to severe chronic pain during one to four years of follow up were calculated using Generalized Estimation Equations (GEE) with robust standard errors. To obtain Relative Risk (RR) as the estimate, a Poisson distribution with a log link was used. We used an exchangeable working correlation structure, assuming similar correlation between the repeated measures. The chosen working correlation structure was compared to an independent structure, assuming no correlation between the repeated measurements, using the Quasi Likelihood under Independence Model Criterion (QIC) for all models. The goodness of fit between the partly adjusted and fully adjusted models were compared using the Corrected Quasi Likelihood under Independence Model Criterion (CQIC). Continuous predictors with an apparent non-linear relationship with the outcome were categorized. The GEE analyses were carried out separately for those reporting no chronic pain and those reporting chronic pain of any severity at baseline, and pain severity (no or mild vs moderate or severe) was used as a predictor variable. Missing data was handled by including all available follow up information. That is, subjects were included if they had responded to at least one follow up measure.

All analyses were carried out using SPSS 23 (IBM Corp. Armonk, NY) and STATA 14 (Stata Corp. College Station, Texas). 


\section{Results}

Among those answering both questions measuring chronic pain at baseline $(N=4694)$, the sex ratio was similar to the invited sample, whereas the proportion of subjects in the youngest age group was somewhat lower (table 1). The participation rate dropped gradually, and at the final occasion 3360 subjects answered both questions. While sex was not associated with drop out, the proportion of subjects in the youngest age group and the proportion of subjects with primary education only, declined throughout the whole period. The prevalence of chronic pain of at least moderate intensity increased from $28 \%$ on the first two occasions to $30 \%$ on the final three occasions (table 1 ).

\subsection{The development and course of chronic pain}

Among 1655 subjects reporting no chronic pain at baseline, 79 had developed chronic pain of at least moderate intensity by one year follow up, giving an annual cumulative incidence of $4.8 \%$ (95\% $\mathrm{Cl}$ : 3.8-5.9). The probability of maintaining the status of at least moderate chronic pain by the second year of follow up was $38.0 \%$ (95\% Cl: $28.1-49.0)$, and thereafter the probability exceeded $60 \%$ on the three- and four year follow up (figure 2). The corresponding proportion reporting mild pain on the first year off follow up, was $11 \%$ (not included in figure 2) whereas $84.2 \%$ (95\% Cl: 82.4 85.9) maintained a status of no chronic pain. When a status of no chronic pain was maintained for several years, the probability of changing to a chronic pain status on the following occasion diminished substantially. Only $1 \%(95 \% \mathrm{Cl}$ : 0.5.1.8) developed moderate to severe chronic pain after having had no chronic pain during the previous 3 measurements.

-Please insert figure2 about here-

Among 833 subjects with moderate to severe chronic pain at baseline, the proportion maintaining that status during the first year of follow up was $70.8 \%(95 \% \mathrm{Cl}: 67.7-73.8)$ whereas $8.2 \%(95 \% \mathrm{Cl}$ : 6.5-10.2) recovered into no chronic pain (figure 3) and $21.0 \%$ transferred to mild chronic pain (not included in figure 3). As the chronic pain persisted, the probability of recovery decreased. Among those reporting at least moderate chronic pain consistently during the previous four measurements, 92.4\% (95\% Cl: 89.4-94.6) still reported it on the final measurement, whereas 3.2\% (95\% $\mathrm{Cl}: 1.9-5.4)$ recovered into no chronic pain (figure 3). The probability of maintaining a no chronic pain status the following year ranged from $38.1 \%$ to $51.5 \%$, but then it increased as the no chronic pain status was prolonged (figure 3). 
-Please insert figure 3 here-

Table 2 gives the number of occasions with moderate to severe chronic pain according to baseline chronic pain status. The majority of subjects who changed from no to moderate or severe chronic pain, had only one occasion. Whereas $62.3 \%$ of those with moderate to severe chronic pain at baseline had at least three of four occasions with moderate to severe chronic pain during follow up. No occasions with chronic pain were reported by 1051 subjects, which amounts to $87.1 \%$ of those without chronic pain at baseline, and $63.5 \%$ of the total sample. In contrast, 376 participants reported moderate to severe chronic pain on all occasions, which amounts to $45.1 \%$ of those with moderate to severe chronic pain at baseline and $11.2 \%$ of the total sample.

-Please insert table 2 here-

\subsection{Baseline predictors of chronic pain during follow up}

Among those without chronic pain at baseline, the probability of reporting moderate to severe chronic pain increased with time in analyses adjusted for sex, age and education ( $\mathrm{RR}=1.17 ; 95 \% \mathrm{Cl}$ : 1.10-1.25) (table 3). No effect of time was seen among those reporting chronic pain at baseline. Female sex, higher age and lower level education were significant predictors of future moderate to severe chronic pain in both partial and fully adjusted analyses, both among those with and those without chronic pain at baseline (Table 3). Being overweight significantly predicted future moderate to severe chronic pain among those not reporting chronic pain at baseline (RR=1.64; 95\% Cl: $1.24-$ 2.17), but less so among those reporting chronic pain at baseline ( $R R=1.04 ; 95 \% \mathrm{Cl}: 0.97-1.12)$ in analyses adjusting for other co-variables. On the other hand, smoking status did not significantly predict future chronic pain among those without chronic pain at baseline, but being a current smoker was significantly associated with a poorer prognosis among those with chronic pain at baseline ( $R R=1.13 ; 95 \% \mathrm{Cl}: 1.05-1.22)$. Reporting at least moderate pain and widespread pain at baseline were also significant predictors of future moderate to severe chronic pain in both groups. Among those without chronic pain at baseline, the association lost statistical significance when also controlling for other predictors in the fully adjusted model (RR=1.30; 95\% $\mathrm{Cl}: 0.79-2.15)$. Pain catastrophizing and dissatisfaction with sleep were both significant predictors of future chronic pain in both partial and fully adjusted analyses, both among subjects with and without chronic pain at 
baseline. An MHI-5 score below cut off indicating depression $(\leq 60)$ was also a significant predictor of future chronic pain in all models, except in the fully adjusted model for those without chronic pain at baseline ( $\mathrm{RR}=1.27$; 95\% $\mathrm{Cl}$ : 0.77-2.09).

-Please insert table 3 here-

The Corrected Quasi Likelihood under Independence Model Criterion (CQIC) indicated that the fully adjusted models obtained the best fit. For subjects without chronic pain at baseline the CQIC was 2483.8 for the fully adjusted model and 2627.4 for the model including age, sex education and time. For subjects with chronic pain at baseline, the corresponding figures were 3505.0 and 4056.1, respectively.

Using an independent correlation structure revealed similar estimates as those obtained with the exchangeable correlation structure. The quasi Likelihood under Independence Model Criterion (QIC) were identical for the two correlation structures $(Q I C=2496.1)$ in the fully adjusted analyses of subjects without chronic pain at baseline, and 3495.3 versus 3495.2 among those with chronic pain at baseline, revealing an equal goodness of fit for the two different correlation structures.

\section{Discussion:}

We followed a population-based sample over four years to investigate the development and course of chronic pain. Among those without chronic pain at baseline, the proportion developing new chronic pain of at least moderate severity during the first year (annual cumulative incidence) was about five percent. However, when a status of no chronic pain was maintained for several years, the probability diminished substantially. For those with moderate to severe chronic pain at baseline, the probability of recovery into no chronic pain during the first year was about eight percent, and then the recovery rate declined as the pain persisted. After having developed a new moderate to severe chronic pain, the probability of maintaining it was $38 \%$, and after having recovered, the probability of maintaining the recovery was $52 \%$.

Annual cumulative incidence rates of $2 \%$ and $8 \%$ and recovery rates of $5 \%$ and $9 \%$ have previously been reported (Elliott et al., 2002; Eriksen et al., 2004). However, these figures are difficult to compare due to differences in case ascertainments and follow up intervals. In contrast to previous studies, we focused on the transitions between no chronic pain and chronic pain of at least moderate severity, excluding minor fluctuations such as changes from no to mild or from mild to moderate chronic pain. Nonetheless, our annual cumulative incidence and recovery estimates are 
within the ranges reported previously, verifying that major changes in chronic pain occur in a notable proportion of the general population.

Our study also adds to previous findings that both cumulative incidence and recovery rates are heavily dependent on previous chronic pain. That is, chronic pain is more likely to persist for subjects with an extensive history of pain. Moreover, the recovery may only be temporary for a large proportion, indicating that chronic pain is a dynamic condition, which many move in and out of. It is therefore difficult to obtain exact cumulative incidence estimates as any study will have a period prior to baseline in which pain has not been measured. This is underscored by the fact that chronic pain is common also among children and adolescents (King et al., 2011) and several factors in childhood and adolescence predicts pain in adulthood (Dunn et al., 2013).

Other studies have used latent class analyses to categorize subjects with different courses of low back pain (Kongsted et al., 2016). These studies tend to show that low back pain may be characterized by both stable and fluctuating courses, and in most studies, a group of subjects who recover. Our results support the notion of classifying subjects with chronic pain based on different courses by showing that individuals with a stable course may be separated from those with a fluctuating course and those with a single episode. Whether this is a feature shared by many different pain conditions, or it is a contribution based on a substantial prevalence of low back pain in the general population (Hoy et al., 2012), should be a matter of future investigation.

Von Korff and Miglioretti (2005) found that the prediction of future chronic pain was significantly improved by adding prognostic markers such as depression and distribution of pain across the body. We included a broad set of risk factors which have previously shown robust evidence for a relationship with chronic pain (Finan et al., 2013; Gerrits et al., 2015; Mallen et al., 2007). They included both demographic and lifestyle factors, pain characteristics and psychological factors. These factors have rarely been included concomitantly in previous studies. We showed that by mutually adjusting for pain severity, widespread pain, pain catastrophizing, depression and sleep problems, all were related to a poor prognoses among those with chronic pain at baseline. This suggests that all these factors are important and considering them collectively may improve our prediction of prognosis. Neither widespread pain nor depression was significantly related to future chronic pain among those without chronic pain at baseline in the fully adjusted model. Widespread pain and depression were both uncommon among those without chronic pain, and this contributed to wide confidence intervals. The tendency was clear however, and both widespread pain and depression were associated with future chronic pain in the partially adjusted model, indicating that they may be important prognostic factors when presented in subjects without chronic pain. 
Some other factors were considered, but not included in the analyses. Evidence for an association between physical activity and future chronic pain is inconsistent, and this relationship may need further investigation in more detailed analyses (Heneweer et al., 2011; Landmark et al., 2013b). Chronic pain may be a symptom of a wide range of chronic diseases or it may be of unknown cause and in many cases a combination of both. We chose not to include a measure of comorbidity as a predictor since it is difficult to determine if a chronic disease is a direct cause or a comorbid condition.

As most longitudinal studies, this study was subject to loss to follow up. From baseline to 4 years follow up the attrition rate was $29 \%$. The male - female ratio was similar at every occasion, whereas the proportion of individuals in the youngest age group declined and the proportion of middle aged individuals increased from baseline to four years follow up. We found a slight increase in the prevalence of chronic pain over the follow up period. Thus, the attrition may have been somewhat higher among subjects without chronic pain. However, this increase in prevalence partly relates to a general aging of the sample. Therefore, chronic pain was not a major determinant for the participation. The sample was randomly drawn from the population based HUNT 3 study, which had a participation rate of 54\%. Participation in the HUNT 3 study was lower among men, among those in the youngest age groups, among those with lower socioeconomic status as well as those without chronic musculoskeletal pain (Langhammer et al., 2012). This may have reduced the generalizability of the current findings. In particular, the incidence rates are likely to be somewhat lower and the recovery rates somewhat higher in a younger population including more men. On the contrary, as our analyses on education suggest, the incidence is likely to be higher and the recovery lower in a population including more members at the lower end of the socioeconomic ladder. Analyses of associations in epidemiological studies have been shown to be less affected by the participation rate then prevalence and incidence estimates (Galea and Tracy 2007). The figures representing predictors of chronic pain during the follow up period may therefore be interpreted with less caution.

The study sample size was determined to be able to estimate incidence estimates as low as $2 \%$ with sufficient precision. Our main results is well beyond this, but estimates that is based on smaller subgroups have to be interpreted more carefully in line with the provided confidence intervals. No formal and standardized definition of chronic pain exists. In the upcoming ICD-11, chronic pain will be defined as persistent or recurrent pain lasting longer than three months (Treede et al., 2015). The proposal also takes into account additional criteria for severity and functioning. Our definition of chronic pain is therefore conceptually similar to this. In a recent meta-analysis, it was shown that variations in prevalence estimates was not related to different duration criteria used to define 
chronic pain in the various studies (Steingrimsdottir et al., 2017). However, other factors such as the phrasing and framing of the question and the choice of additional criteria may influence the classification. As there are no objective measures or gold standard for detecting chronic pain, there will be uncertainty associated with the classification. Future research will benefit from agreeing on standardized measures.

\section{Conclusion:}

This study gives further evidence for the major health challenge chronic pain poses to society by demonstrating that it is a lasting problem for many. For others, chronic pain is a condition they move in and out of. Chronic pain should be viewed in the context of previous experiences of pain. Clinical evaluation of patients with chronic pain is likely to improve by undertaking a thorough case history including the course and intensity of the chronic pain. When also considering additional prognostic factors, one may improve patient classification and thereby patient information and communication. This is particularly important when discussing with the patient whether recovery is a realistic goal. When recovery seems unrealistic, treatment may focus on other aspects to relieve suffering and improve functioning and health. It is also important to identify and target modifiable prognostic factors such as poor sleep, depression and catastrophizing at an early stage. Treatment focused on these factors may increase the probability of recovery, particularly in newly developed chronic pain.

Acknowledgements: The Nord-Trøndelag Health Study (The HUNT Study) is a collaboration between HUNT Research Centre (Faculty of Medicine, Norwegian University of Science and Technology, NTNU), Nord-Trøndelag County Council, Central Norway Health Authority, and the Norwegian Institute of Public Health.

Author Contributions: The study was planned, designed and conducted by TL, OD, PCB, PR and SK. TL conducted the statistical analyses and wrote the paper. All authors discussed the results and commented on the manuscript. 


\section{References:}

Berwick DM, Murphy JM, Goldman PA, Ware JE, Jr., Barsky AJ, Weinstein MC. Performance of a fiveitem mental health screening test. Med Care 1991;29: 169-176.

Carnes D, Parsons S, Ashby D, Breen A, Foster NE, Pincus T, Vogel S, Underwood M. Chronic musculoskeletal pain rarely presents in a single body site: results from a UK population study. Rheumatology (Oxford) 2007;46: 1168-1170.

Chapman CR, Tuckett RP, Song CW. Pain and stress in a systems perspective: reciprocal neural, endocrine, and immune interactions. J Pain 2008;9: 122-145.

Choi Y, Mayer TG, Williams MJ, Gatchel RJ. What is the best screening test for depression in chronic spinal pain patients? The Spine Journal 2014;14: 1175-1182.

Dunn KM, Hestbaek L, Cassidy JD. Low back pain across the life course. Best Pract Res Clin Rheumatol 2013;27: 591-600.

Elliott AM, Smith BH, Hannaford PC, Smith WC, Chambers WA. The course of chronic pain in the community: results of a 4-year follow-up study. Pain 2002;99: 299-307.

Eriksen J, Ekholm O, Sjogren P, Rasmussen NK. Development of and recovery from long-term pain. A 6-year follow-up study of a cross-section of the adult Danish population. Pain 2004;108: 154162.

Finan PH, Goodin BR, Smith MT. The association of sleep and pain: an update and a path forward. J Pain 2013;14: 1539-1552.

Galea Sand Tracy M. Participation Rates in Epidemiologic Studies. Ann Epidemiol 2007;17: 643-653.

Gerrits MMJG, van Marwijk HWJ, van Oppen P, van der Horst H, Penninx BWJH. Longitudinal association between pain, and depression and anxiety over four years. J Psychosom Res 2015;78: 64-70.

Heneweer H, Staes F, Aufdemkampe G, van Rijn M, Vanhees L. Physical activity and low back pain: a systematic review of recent literature. Eur Spine J 2011;20: 826-845.

Hoy D, Bain C, Williams G, March L, Brooks P, Blyth F, Woolf A, Vos T, Buchbinder R. A systematic review of the global prevalence of low back pain. Arthritis Rheum 2012;64: 2028-2037.

Jensen MK, Sjøgren P, Ekholm O, Rasmussen NK, Eriksen J. Identifying a long-term/chronic, noncancer pain population using a one-dimensional verbal pain rating scale: an epidemiological study. European Journal of Pain 2004;8: 145-152.

Jensen MP, Keefe FJ, Lefebvre JC, Romano JM, Turner JA. One- and two-item measures of pain beliefs and coping strategies. Pain 2003;104: 453-469.

Kamaleri Y, Natvig B, Ihlebaek CM, Bruusgaard D. Localized or widespread musculoskeletal pain: does it matter? Pain 2008;138: 41-46.

King S, Chambers CT, Huguet A, MacNevin RC, McGrath PJ, Parker L, MacDonald AJ. The epidemiology of chronic pain in children and adolescents revisited: a systematic review. Pain 2011;152: 2729-2738.

Kongsted A, Kent P, Axen I, Downie AS, Dunn KM. What have we learned from ten years of trajectory research in low back pain? BMC Musculoskeletal Disorders 2016;17: 220.

Krokstad S, Langhammer A, Hveem K, Holmen TL, Midthjell K, Stene TR, Bratberg G, Heggland J, Holmen J. Cohort Profile: the HUNT Study, Norway. Int J Epidemiol 2013;42: 968-977.

Landmark T, Romundstad P, Dale O, Borchgrevink PC, Kaasa S. Estimating the prevalence of chronic pain: Validation of recall against longitudinal reporting (the HUNT pain study). Pain 2012;153: 1368-1373.

Landmark T, Romundstad P, Dale O, Borchgrevink PC, Vatten L, Kaasa S. Chronic pain: One year prevalence and associated characteristics (the HUNT pain study). Scandinavian Journal of Pain 2013a;4: 182-187. 
Landmark T, Romundstad PR, Borchgrevink PC, Kaasa S, Dale O. Longitudinal associations between exercise and pain in the general population--the HUNT pain study. PloS one 2013b;8: e65279.

Langhammer A, Krokstad S, Romundstad P, Heggland J, Holmen J. The HUNT study: participation is associated with survival and depends on socioeconomic status, diseases and symptoms. BMC Medical Research Methodology 2012;12: 143.

Mallen CD, Peat G, Thomas E, Dunn KM, Croft PR. Prognostic factors for musculoskeletal pain in primary care: a systematic review. Br J Gen Pract 2007;57: 655-661.

Merskey Hand Bogduk N. Classification of chronic pain: descriptions of chronic pain syndroms and definitions of pain terms. Seatlle, WA: IASP Press. 1994.

Ohayon MMand Paiva T. Global sleep dissatisfaction for the assessment of insomnia severity in the general population of Portugal. Sleep Med 2005;6: 435-441.

Rumpf H-J, Meyer C, Hapke U, John U. Screening for mental health: validity of the MHI-5 using DSMIV Axis I psychiatric disorders as gold standard. Psychiatry Res 2001;105: 243-253.

Schmidt COand Baumeister SE. Simple patterns behind complex spatial pain reporting? Assessing a classification of multisite pain reporting in the general population. Pain 2007;133: 174-182.

Steingrimsdottir OA, Landmark T, Macfarlane GJ, Nielsen CS. Defining chronic pain in epidemiological studies: a systematic review and meta-analysis. Pain 2017;158: 2092-2107.

Treede RD, Rief W, Barke A, Aziz Q, Bennett MI, Benoliel R, Cohen M, Evers S, Finnerup NB, First MB, Giamberardino MA, Kaasa S, Kosek E, Lavand'homme P, Nicholas M, Perrot S, Scholz J, Schug S, Smith BH, Svensson P, Vlaeyen JW, Wang SJ. A classification of chronic pain for ICD-11. Pain 2015;156: 1003-1007.

Von Korff Mand Miglioretti DL. A prognostic approach to defining chronic pain. Pain 2005;117: 304313.

Ware JE, Kosinski M, Dewey JE, Gandek B. How to score and interpret single item health status measures: A manual for users of the SF-8 health Survey. Lincoln: Quality Metric Incorporated. 2001.

Williams F, Spector T, MacGregor A. Pain reporting at different body sites is explained by a single underlying genetic factor. Rheumatology (Oxford) 2010;49: 1753-1755.

Wolfe F, Egloff N, Hauser W. Widespread Pain and Low Widespread Pain Index Scores among Fibromyalgia-positive Cases Assessed with the 2010/2011 Fibromyalgia Criteria. J Rheumatol 2016;43: 1743-1748.

Wolfe F, Smythe HA, Yunus MB, Bennett RM, Bombardier C, Goldenberg DL, Tugwell P, Campbell SM, Abeles M, Clark P. The American College of Rheumatology 1990 Criteria for the Classification of Fibromyalgia. Report of the Multicenter Criteria Committee. Arthritis Rheum 1990;33: 160-172. 


\section{Legends}

Figure 1: Flow of participants through the study and the analyses

Figure2: Year by year probability (\%) of maintaining a status of no chronic pain (stability) and of transition into chronic pain (annual cumulative incidence) according to the stability

Figure 3: Year by year probability (\%) of maintaining a status of at least moderate chronic pain (stability) and of transition into no chronic pain (recovery) according to the stability

Table 1: Demographic characteristics and prevalence of chronic pain at the five annual follow up measures of the HUNT pain study

Table 2: Number of occasions with moderate to severe chronic pain from one to four years of follow up according to chronic pain status at baseline

Table 3: Associations between baseline characteristics and moderate to severe chronic pain during one to four years of follow up among subjects not reporting and subjects reporting chronic pain at baseline 
Development and course of chronic pain 16 